\title{
Traduire
}

Une autre perspective sur r tr traduction

Revue française de la traduction

$235 \mid 2016$

Luxe, mode... et traduction !

\section{Créer du rêve : trois leviers pour mieux traduire le luxe}

Agnès Portais

\section{OpenEdition}

1 Journals

Édition électronique

URL : https://journals.openedition.org/traduire/831

DOI : $10.4000 /$ traduire.831

ISSN : 2272-9992

Éditeur

Société française des traducteurs

Édition imprimée

Date de publication : 1 décembre 2016

Pagination : 12-16

ISSN : 0395-773X

\section{Référence électronique}

Agnès Portais, "Créer du rêve : trois leviers pour mieux traduire le luxe », Traduire [En ligne], 235 | 2016,

mis en ligne le 01 décembre 2018, consulté le 07 juillet 2021. URL : http://journals.openedition.org/ traduire/831 ; DOI : https://doi.org/10.4000/traduire.831 


\section{Créer du rêve : \\ trois leviers pour mieux traduire le luxe}

\section{Agnès Portais}

Traductrice indépendante spécialisée dans le domaine du luxe, de la mode et de la beauté, je suis familière des défis à relever avec les mots du rêve. Évoquer la taille poire d'une émeraude en joaillerie ou le détail d'un mécanisme de montre en horlogerie, décrire la pyramide olfactive d'une fragrance orientale ou chyprée, souligner le velouté d'une ombre à paupières, mentionner une coupe, un tombé, une broderie ou une emmanchure sous les lumières d'un créateur et de maisons de couture réputés, expliquer les effets anti-âge d'un sérum hydratant à la sensorialité envoûtante...

Traduire le luxe, c'est écrire les mots de la beauté et de l'élégance en s'appuyant sur l'image d'une marque, l'esprit d'une maison et un style marketing recherché, tout en prenant possession d'un rêve, d'une émotion et d'un désir d'évasion.

Trois leviers différents me semblent indispensables pour bien communiquer dans cet univers du luxe : maîtriser les codes stylistiques d'une marque, restituer le caractère poétique d'un texte dans la langue cible et évoluer avec les nouvelles formes rédactionnelles.

\section{1 - L'identité stylistique d'une marque}

Écrire ou traduire pour le monde du luxe exige d'en maîtriser les codes et donc de se documenter sans cesse, car les nouveautés lancées au rythme des saisons et des évènements sont parfois des innovations technologiques, souvent des créations de designers, et toujours des objets fascinants.

Maîtriser la terminologie d'un secteur ou d'une activité est une chose, mais connaître et savoir reproduire le style rédactionnel d'une marque donnée en est une autre.

Lors d'une de mes premières expériences professionnelles, j'ai été confrontée à un questionnement terminologique. Ce moment m'est resté comme fondateur dans la compréhension de la traduction du luxe. En effet, c'était la première fois que l'on m'expliquait que plusieurs termes 
à l'apparence synonymes pouvaient être utilisés uniquement par une marque et non par une autre. C'était une révélation. II s'agissait des termes " légendaire ", " mythique " et « iconique ". Bien sûr, il existe des termes équivalents en anglais, legendary, mythic/mythical et iconic, mais ce que j'apprenais, c'était que l'un ne traduisait pas forcément l'autre. "Légendaire " convient davantage à une maison de luxe très ancienne, avec une histoire et un patrimoine précieux, et à l'élégance raffinée. "Mythique " renvoie une image un peu pompeuse et convient mieux à une marque qui veut faire sensation, assume une histoire mais veut aussi paraître moderne. "Iconique " donne un ton dynamique, énergique et jeune. C'est un mot qui a pris de l'ampleur ces dernières années. Chacun de ces mots peut être associé à une marque précise et les intervertir ferait perdre complètement l'esprit du texte. En anglais, ces significations peuvent être complètement différentes. Legendary ne se traduira donc pas par "légendaire " si la marque en question se veut moderne et dynamique.

Le texte, qui se doit de refléter au mieux le style rédactionnel d'une marque, est sa signature publicitaire. Dans le secteur du luxe, peu de marques communiquent avec un slogan. Le seul nom de la maison suffit la plupart du temps à évoquer raffinement, produits de qualité ou allure élégante. Récemment, une marque Beauté a adopté une accroche publicitaire en français et en anglais. Le slogan s'adresse directement au client potentiel en l'interpellant par le pronom personnel "vous/you ". Un point important pour une marque qui place le service client au cœur de ses valeurs. En anglais, la formule est joliment poétique, et le style fluide et élégant. En français, en revanche, avec un style trop oral la phrase peut manquer de préciosité, de luxe et peut même paraître peu soignée. D'où une traduction qui avait beau être parfaite au niveau du sens mais qui, pour moi, n'avait pas forcément respecté l'identité de la marque.

La veille documentaire fait donc partie de mon quotidien. Bien connaître l'histoire des grandes maisons du luxe est essentiel (surtout pour celles qui ont un fort patrimoine). S'informer de ce que font les marques concurrentes également. Un des aspects majeurs de la traduction du luxe est également celui de savoir évoquer la technicité des savoir-faire. De plus, le secteur est sans cesse en mouvement et les lancements de produit s'enchaînent à un rythme effréné.

Pour bien traduire un texte, le traducteur doit s'imprégner de toute l'identité de la marque. Le luxe est un domaine qui affiche de fortes disparités de style rédactionnel entre les marques même si, à première vue, les produits ciblent la même clientèle. La difficulté est de prendre le temps de bien se documenter en amont et tout au long d'une collaboration.

\section{2 - La poésie d'une langue cible}

Le luxe est un domaine de traduction qui affectionne tout particulièrement les belles formules de la langue française. Le traducteur, tout comme le rédacteur, doit trouver les mots justes pour transcrire un rêve et susciter une émotion. 
Dans une campagne de publicité récente, une grande marque de luxe avait décliné son annonce presse en pas moins de trois langues. En français, en anglais ou en allemand, le texte se devait de véhiculer la même cohérence que le visuel, qui évoquait une idée de "flânerie ".

J'ai trouvé que cette campagne était parfaitement réussie car elle incarnait - même dans son discours - tout ce que la marque représente et souhaite transmettre : la poésie, la lumière, l'élégance, la beauté du geste et des objets, le temps qui passe, le plaisir des petites choses, les couleurs, la finesse d'une matière, le souci du détail. . .

Cette campagne était un remarquable exemple d'adaptation (ou transcréation). En anglais comme en allemand, on utilisait le terme français "flâner " ou bien un dérivé issu du français. Je me suis demandé comment cela était perçu par les lecteurs cibles. L'usage du français apporte, semble-t-il, une touche particulière et une idée du luxe à la française.

En français : "Dans l'œil du flâneur ". Charles Baudelaire, l'un des plus grands poètes français, aurait contribué à faire évoluer le sens de "flâneur " et à lui apporter une touche légèrement romantique.

En anglais : "Flâneur forever ". Le terme " flâneur " apparaît. II est plutôt rare en anglais. II a un usage essentiellement littéraire. On imagine un dandy anglais dans les rues de Londres. Ici sont soulignés l'intemporalité du style, le savoir-faire précieux d'une marque de luxe qui prend son temps pour imaginer ses collections. Un attribut du raffinement et de la sophistication, mis en exergue ici avec finesse.

En allemand : "Flanieren mit Hermès ". C'est le terme flanieren qui est utilisé. II s'agit d'un emprunt du français dont voici la définition selon le dictionnaire Duden : " éty. Fr. Se promener sans but précis ". L'allemand est la seule version de la publicité qui utilise le nom de la marque dans la signature. Un moyen d'associer l'élégance et le mystère du message à un élément familier. Le ton est donné.

Le thème de la campagne correspond à l'ADN de la marque, l'image du flâneur est positive aussi bien en français qu'en anglais et en allemand. Et surtout la marque tire son épingle du jeu en utilisant la poésie de la langue française pour séduire et faire rêver.

\section{3 - Les nouvelles formes rédactionnelles}

Le hashtag (ou mot-dièse) est apparu il y a quelques années seulement. Ce sont ces petits mots précédés d'un dièse sur un fil Facebook ou Twitter ou bien sur les bandeaux des émissions de télévision. De plus en plus confrontée à cette nouvelle forme rédactionnelle, je me suis posé la question de savoir, en tant que traductrice, s'il fallait ou non les traduire. Incongrue au premier abord, cette problématique soulève à la fois des questions juridiques, culturelles et terminolo- 
giques. Les grandes marques internationales du luxe ont largement recours au hashtag pour relayer leurs campagnes sur les réseaux sociaux.

Depuis le début de son utilisation, le hashtag n'est la plupart du temps pas traduit du tout de l'anglais.

Que dit la loi à ce sujet? En France, tout message publicitaire doit être traduit. C'est la loi Toubon de mars 1994 qui définit l'usage de la langue française sur les supports marketing et publicitaires, ainsi que les obligations des annonceurs à cet égard. Voici ce qu'elle précise :

\section{Article 2}

Dans la désignation, l'offre, la présentation, le mode d'emploi ou d'utilisation, la description de l'étendue et des conditions de garantie d'un bien, d'un produit ou d'un service, ainsi que dans les factures et quittances, l'emploi de la langue française est obligatoire.

Les mêmes dispositions s'appliquent à toute publicité écrite, parlée ou audiovisuelle. Les dispositions du présent article ne sont pas applicables à la dénomination des produits typiques et spécialités d'appellation étrangère connus du plus large public.

La législation sur les marques ne fait pas obstacle à l'application des premier et troisième alinéas du présent article aux mentions et messages enregistrés avec la marque.

Une fois ce point éclairci, la question de la traduction se pose donc pour le marché français. Après l'étude de plusieurs exemples publicitaires de marques de luxe, voici ce qu'il en ressort :

- Pour les marques qui décident de traduire, la traduction doit-elle être littérale ? L'anglais est une langue parfaitement adaptée au hashtag, formule courte et percutante. Mais si on envisage une traduction littérale, le français est souvent bancal, trop long et beaucoup moins efficace.

- Pour le marché français, la traduction peut apparaître en tout petit sur le côté : elle semble la plupart du temps un peu longue et peu percutante. La traduction est placée au même endroit que les mentions légales. Inscrit en tout petit, le texte en français est moins accrocheur, de toute évidence.

- L’Allemagne n'a pas la même politique linguistique, ni la même réglementation au niveau de la publicité. Une marque considère donc qu'en s'exprimant en anglais, elle peut atteindre de la même façon la clientèle cible d'un parfum, par exemple. Vu la complexité de la langue allemande, la traduction du hashtag peut être un vrai défi pour obtenir un message d'un même niveau d'efficacité.

Dans le cas de marques de luxe françaises qui utilisent le hashtag, comment perçoit-on en Allemagne leur communication publicitaire essentiellement en anglais ? Je peux supposer que laisser un texte en anglais apporte une touche de modernité pour les lecteurs allemands. 
Mais un texte en français n'apporterait-il pas un supplément de chic et d'élégance, en cohérence avec l'esprit de la maison de luxe?

- Certaines marques choisissent non pas de traduire le hashtag, mais de l'adapter en français. Même si le texte est inscrit en tout petit sur le côté (avec un astérisque renvoyant vers le hashtag en anglais), la marque opte pour un texte fluide, aussi percutant dans la langue cible que dans la langue source. On parle alors de transcréation.

En résumé, les marques internationales choisissent la plupart du temps de créer un hashtag en anglais pour leur communication marketing déclinée dans le monde entier. Puis, selon les marques et les réglementations, elles font le choix soit de ne rien traduire, soit de le traduire un peu maladroitement, soit de l'adapter au contexte culturel et linguistique.

Personnellement, j'aurais tendance à penser que les marques de luxe françaises pourraient être gagnantes à utiliser le hashtag en français dans leur communication pour transmettre une idée d'élégance et afficher la beauté de la langue française dans le monde entier.

\section{Conclusion}

Les facettes du métier de traducteur spécialisé dans le luxe sont nombreuses. J'écris et traduis selon un style d'écriture respectant une signature rédactionnelle, un ADN lexical et terminologique précis. Dans mon métier au quotidien, je joue avec la beauté de la langue française. La communication marketing actuelle nécessite une expertise à la fois des pleins et des déliés d'une langue, du storytelling, mais aussi d'une connaissance approfondie de l'objet du discours. Le "Luxe " est un domaine où les mots se doivent d'être choisis, où le style mérite d'être fluide et où la formule peut constituer un véritable vecteur du rêve. Voilà précisément ce qui fait la difficulté et le charme de ce métier.

agnes@poudreauxyeux.fr

Fondatrice de Poudre aux yeux, Agnès Portais est rédactrice et traductrice freelance. Elle a créé sa société en 2011 à la suite de plusieurs expériences chez l'annonceur et en agences. Parisienne d'adoption, elle a toujours eu le goût des mots et l'envie de regarder au-delà des frontières. Après un parcours scolaire et universitaire littéraire et linguistique (Hypokhâgne, ISIT), ses expériences professionnelles la mènent dans le milieu du luxe, de la mode et de la beauté. Elle imagine alors Poudre aux yeux pour mieux passer d'une langue à l'autre, d'une culture à l'autre, d'une idée à des mots dont le sens est porteur de rêves. Avec l'anglais, l'allemand et le français comme langues de travail, elle rédige, traduit et, depuis quelque temps, partage également son expérience de traductrice et de rédactrice Luxe dans un blog intitulé Des mots du rêve : www.poudreauxyeux.fr/blog 